International Journal on Cybernetics \& Informatics (IJCI) Vol. 4, No. 1, February 2015

\title{
Maneuvering TARget Track PREDiCtion MODEL
}

\author{
Qingping $\mathrm{Yu}^{1}$, Xiaoming $\mathrm{You}^{2}$ and Sheng Liu ${ }^{3}$ \\ ${ }^{1}$ College of Electronic and Electrical Engineering, Shanghai University of Engineering \\ Science, Shanghai 201620, China \\ ${ }^{2}$ School of Management, Shanghai University of Engineering Science \\ Shanghai, 201620, China
}

\begin{abstract}
The issues about maneuvering target track prediction were discussed in this paper. Firstly, using Kalman filter which based on current statistical model describes the state of maneuvering target motion, thereby analyzing time range of the target maneuvering occurred. Then, predict the target trajectory in real time by the improved gray prediction model. Finally, residual test and posterior variance test model accuracy, model accuracy is accurate.
\end{abstract}

\section{KEYWORDS}

Current Statistical Model, Maneuvering Target, Kalman Filter, Gray Theory

\section{THE STATE DESCRIPTION OF MANEUVERING TARGET}

To build model of maneuvering target motion by current statistical model, to use Kalman filter process tracking.

\subsection{To build current statistical model for maneuvering target}

Current statistical model is a nonzero mean time-dependent model. Current acceleration probability density is described by the modified Rayleigh distribution. It is assumed that the target acceleration $a(\mathrm{t})$ satisfies the following relationship:

$$
\begin{aligned}
& a(\mathrm{t})=\bar{a}(\mathrm{t})+a_{1}(\mathrm{t}) \\
& a_{1}^{\prime}(\mathrm{t})=-\alpha a_{1}(\mathrm{t})+\omega(\mathrm{t})
\end{aligned}
$$

In the above formula: $\bar{a}$ is the mean acceleration, and it is an estimate (a constant in each sampling period) about previous time acceleration; ${ }^{a_{1}(\mathrm{t})}$ is a first order Markov process of zero mean, $\alpha$ is the reciprocal of the maneuvering time constant; $\omega(\mathrm{t})$ is zero mean Gaussian white noise.

The discrete state equation of current statistical model is:

DOI: 10.5121/ijci.2015.4104 


$$
X(\mathrm{k}+1)=\mathrm{F}(\mathrm{k}) \mathrm{X}(\mathrm{k})+\mathrm{U}(\mathrm{k}) \bar{a}(\mathrm{k}+1)+\mathrm{W}(\mathrm{k})
$$

$X(k)$ is state vector, $F(k)$ is state transition matrix, $\mathrm{U}(k)$ is Disturbance transfer matrix , $\mathrm{W}(k)$ is zero mean white noise with variance $Q(k)$, radar scan for 1 s, so the sampling period $T=1$, take $\alpha=1$, the following expression:

$$
\begin{aligned}
& X(\mathrm{k})=\left[x(\mathrm{k}) \quad x^{\prime}(\mathrm{k}) \quad x^{\prime \prime}(\mathrm{k})\right] \\
& F(k)=\left[\begin{array}{ccc}
1 & T & \left(-1+\alpha T+e^{-\alpha T}\right) / \alpha^{2} \\
0 & 1 & \left(1-e^{-\alpha T}\right) / \alpha \\
0 & 0 & e^{-\alpha T}
\end{array}\right] \\
& \mathrm{U}(k)=\left[\begin{array}{c}
\left(-T+\alpha T^{2} / 2+1-\left(\mathrm{e}^{-\alpha T}\right) / \alpha\right) / \alpha \\
T-\left(1-\mathrm{e}^{-\alpha T}\right) / \alpha \\
1-\mathrm{e}^{-\alpha T}
\end{array}\right]
\end{aligned}
$$

In the above formula, $\mathrm{T}$ is the sampling period.

Variance adaptive algorithm based on current statistical model, that is, the following equation adaptive variance:

$$
\mathrm{Q}(\mathrm{k})=\mathrm{E}\left[\mathrm{W}(\mathrm{k}) \mathrm{W}^{T}(\mathrm{k})\right]=2 \alpha \sigma_{a}^{2}\left[\begin{array}{lll}
q_{11} & q_{12} & q_{13} \\
q_{12} & q_{22} & q_{23} \\
q_{13} & q_{23} & q_{33}
\end{array}\right]
$$

The variables are as follows:

$$
\begin{aligned}
& q_{11}=\left[1-e^{-2 \alpha T}+2 \alpha \mathrm{T}+2 \alpha^{3} \mathrm{~T}^{3} / 3-2 \alpha^{2} \mathrm{~T}^{2}-4 \alpha T e^{-\alpha T}\right] / 2 \alpha^{5} \\
& q_{12}=\left[1+\mathrm{e}^{-2 \alpha T}-2 e^{-\alpha T}+2 \alpha \mathrm{Te}^{-\alpha T}-2 \alpha \mathrm{T}+\alpha^{2} \mathrm{~T}^{2}\right] / 2 \alpha^{4} \\
& q_{13}=\left[1-\mathrm{e}^{-2 \alpha T}-2 \alpha \mathrm{Te}^{-\alpha T}\right] / 2 \alpha^{3} \\
& q_{22}=\left[4 e^{-\alpha T}-3-\mathrm{e}^{-2 \alpha T}+2 \alpha \mathrm{T}\right] / 2 \alpha^{3} \\
& q_{23}=\left[e^{-2 \alpha T}+1-2 \mathrm{e}^{-\alpha T}\right] / 2 \alpha^{2} \\
& q_{33}=\left[1-\mathrm{e}^{-2 \alpha T}\right] / 2 \alpha
\end{aligned}
$$




$$
\sigma_{a}^{2}= \begin{cases}\frac{4-\pi}{\pi}\left[\mathrm{a}_{\max }-\bar{a}(\mathrm{k})\right]^{2} & \bar{a}(\mathrm{k})>0 \\ \frac{4-\pi}{\pi}\left[\mathrm{a}_{-\max }-\bar{a}(\mathrm{k})\right]^{2} & \bar{a}(\mathrm{k})<0\end{cases}
$$

$\mathrm{a}_{\max }$ is the maximum positive acceleration, $\mathrm{a}_{-\max }$ is the maximum negative acceleration.

Measurement equation :

$$
Z(\mathrm{k})=\mathrm{H}(\mathrm{k}) \mathrm{X}(\mathrm{k})+\mathrm{V}(\mathrm{k})
$$

In the above formula, $\mathrm{H}(\mathrm{k})$ is measurement matrix, $\mathrm{V}(\mathrm{k})$ is white noise that it's zero mean and variance is $R(\mathrm{k})$ 。

\subsection{Kalman filter based on current statistical model}

Kalman filter based on current statistical model smooth the state of the target on the past and present time, while predicting the target movement in the future time, including the location of the target, velocity and acceleration parameters.

State variable method is a valuable method to describe a dynamic system, using this methods, the system input-output relationship is described in the time domain by the state transition model and output observation model. Input can be determined by dynamic model consisted of a function of time and unpredictable variables or random noise process to describe. Output is a function of the state, which often disturbed by random observation errors, can be described by measurement equation.

Dynamic equations of discrete-time systems (state equations) can be expressed as :

$$
X(\mathrm{k})=F(\mathrm{~K}) \mathrm{X}(\mathrm{k}-1)+\bar{a}(\mathrm{k}) U(\mathrm{k})+\mathrm{W}(\mathrm{k})
$$

The measurement equation of discrete-time systems :

$$
\mathrm{Z}(\mathrm{k})=H(\mathrm{k}) \mathrm{X}(\mathrm{k})+V(\mathrm{k})
$$

$H(\mathrm{k})$ is the measurement matrix,$V(\mathrm{k})$ is zero mean Gaussian white noise sequence. Different time measurement noise is independent

The formulas of Kalman filter algorithm based on the current statistical model are :

$$
\begin{aligned}
& X(\mathrm{k} \mid \mathrm{k}-1)=F(\mathrm{k}) \mathrm{X}(\mathrm{k}-1 \mid \mathrm{k}-1)+\bar{a}(\mathrm{k}) B(\mathrm{k}) \\
& \mathrm{P}(\mathrm{k} \mid \mathrm{k}-1)=F(\mathrm{k}) \mathrm{P}(\mathrm{k}-1 \mid \mathrm{k}-1) \mathrm{F}^{T}(\mathrm{k})+Q
\end{aligned}
$$




$$
\begin{gathered}
\mathrm{X}(\mathrm{k} \mid \mathrm{k})=X(\mathrm{k} \mid \mathrm{k}-1)+K_{g}(\mathrm{k})[\mathrm{Z}(\mathrm{k})-H(\mathrm{k}) \mathrm{X}(\mathrm{k} \mid \mathrm{k}-1)] \\
K_{g}(\mathrm{k})=\mathrm{P}(\mathrm{k} \mid \mathrm{k}-1) \mathrm{H}^{T}(\mathrm{k})\left[\left(\mathrm{H}(\mathrm{k}) \mathrm{P}(\mathrm{k} \mid \mathrm{k}-1) \mathrm{H}^{T}(\mathrm{k})+R(\mathrm{k})\right]^{-1}\right. \\
\mathrm{P}(\mathrm{k} \mid \mathrm{k})=\left[\mathrm{I}-\mathrm{K}_{g}(\mathrm{k}) \mathrm{H}(\mathrm{k})\right] \mathrm{P}(\mathrm{k} \mid \mathrm{k}-1)
\end{gathered}
$$

Wherein, $W(\mathrm{k}) \sim \mathrm{N}(0, Q), \mathrm{V}(\mathrm{k}) \sim \mathrm{N}(0, \mathrm{R})$.

Kalman filter does not require saving measurement data in the past, when new data measured, according to new data and various valuation in previous time, by means of state transition equation of the system, according to the above recurrence formula, thereby calculating the amount of all the new valuation.

\section{TRACK PREDICTION BASED ON THE GRAY THEORY}

To solve track forecast problem of aerial target, an aerial targets track prediction method based on gray theory is proposed in this paper. Grey system theory is based on new understanding about objective system. Although insufficient information on some systems, but as a system must have specific functions and order, but its inherent laws are not fully exposed. Some random amount, no rules interference component and chaotic data columns, from gray system point of view, is not considered to be elusive. Conversely, in gray system theory, a random amount is regard as a gray amount within a certain range, according to the proper way to deal with the raw data, grey number is converted to generation number, and then get strong regularity generation functions from generation number.

To achieve a real-time online track prediction and improve the prediction accuracy, an improved GM forecasting model is established. In the case of limited data, the model is used to predict the track effectively. The basic steps of aerial target track grey prediction: 1) the raw data accumulated generating $1 ; 2)$ the establishment of $\mathrm{GM}(1,1)$ prediction model; 3$)$ to test the model; 4) establishment of a residual model; 5) target track forecast.

\subsection{Target track prediction}

The current track graphics, coordinate information is known.

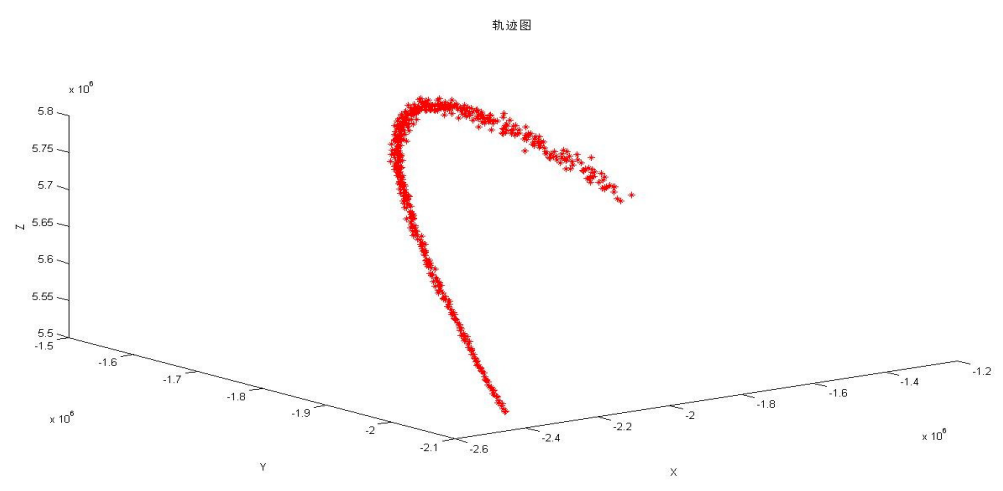

Figure 1. The current track data 
Provided the original data sequence :

$$
\begin{aligned}
& X^{(0)}=\left(\mathrm{x}^{(0)}(1), \mathrm{x}^{(0)}(2), \ldots, \mathrm{x}^{(0)}(\mathrm{n})\right), Y^{(0)}=\left(\mathrm{y}^{(0)}(1), \mathrm{y}^{(0)}(2), \ldots, \mathrm{y}^{(0)}(\mathrm{n})\right) \\
& Z^{(0)}=\left(\mathrm{z}^{(0)}(1), \mathrm{z}^{(0)}(2), \ldots, \mathrm{z}^{(0)}(\mathrm{n})\right),\left(\mathrm{x}^{(0)}(\mathrm{i}), \mathrm{y}^{(0)}(\mathrm{i}), \mathrm{z}^{(0)}(\mathrm{i})\right) \text { is the coordinates of the target }
\end{aligned}
$$

(Figure1 data) in rectangular coordinate system with the center of earth. ${ }^{(0)}(i)>0, i=1,2, \ldots \mathrm{m}$ $y^{(0)}(\mathrm{i})>0, \mathrm{i}=1,2, \ldots \mathrm{m}, z^{(0)}(\mathrm{i})>0, \mathrm{i}=1,2, \ldots \mathrm{m}$, using the data sequence, general procedures to establish GM $(1,1)$ model is :

The first step: to do the first order accumulated generating of the original data series $X^{(0)}$ (i.e., 1 AGO), get accumulated generating sequence:

$$
\begin{gathered}
X^{(1)}=\left(\mathrm{x}^{(1)}(1), \mathrm{x}^{(1)}(2), \ldots, \mathrm{x}^{(1)}(\mathrm{n})\right) \\
\mathrm{x}^{(1)}(1)=\mathrm{x}^{(0)}(1), \mathrm{x}^{(1)}(\mathrm{k})=\sum_{i=1}^{k} \mathrm{x}^{(0)}(\mathrm{i}),(\mathrm{k}=2,3, \ldots, \mathrm{n})
\end{gathered}
$$

Wherein,

The second step: the first order accumulated generating sequence $X^{(1)}$ established GM $(1,1)$ model, getting related albino differential equations:

$$
\frac{d x^{(1)}(\mathrm{t})}{d t}+a x^{(1)}(\mathrm{t})=\mathrm{b}
$$

Where, a is the development factor, reflecting the development trend between $\hat{x}^{(1)}$ and $\hat{x}^{(0)}$. B is gray action , the gray action of GM $(1,1)$ model is mined from the background data, it reflects the relationship to data changes, so the exact meaning is gray.

The related gray differential equation form as:

$$
x^{(0)}(\mathrm{k})+a d^{(1)}(\mathrm{k})=\mathrm{b}, \mathrm{k}=2,3, \ldots
$$

The third step: Solving the parameters a, b. Parameter sequence

$$
B=\left[\begin{array}{cc}
-d^{(1)}(2) & 1 \\
-d^{(1)}(3) & 1 \\
\vdots & \vdots \\
-d^{(1)}(\mathrm{n}) & 1
\end{array}\right]_{\text {can be }}
$$
determined by the least squares method : $\Phi=\left[\mathrm{B}^{T}, \mathrm{~B}\right]^{-1} B^{T} A$, 


$$
B=\left[\begin{array}{cc}
-d^{(1)}(2) & 1 \\
-d^{(1)}(3) & 1 \\
\vdots & \vdots \\
-d^{(1)}(\mathrm{n}) & 1
\end{array}\right], d^{(1)}(\mathrm{k})=\frac{1}{2}\left[\mathrm{x}^{(1)}(\mathrm{k})+\mathrm{x}^{(1)}(\mathrm{k}-1)\right], A=\left(\mathrm{x}^{(0)}(2), \mathrm{x}^{(0)}(3), \ldots, \mathrm{x}^{(0)}(\mathrm{n})\right)^{T} \text { 。 }
$$

The fourth step: In the initial conditions $\hat{x}^{(1)}(1)=x^{(1)}(1)=x^{(0)}(1)$, Generate data series models are available:

$$
\hat{\mathrm{x}}^{(1)}(\mathrm{k})=\left(\mathrm{x}^{(0)}(1)-\frac{\hat{b}}{\hat{a}}\right) e^{-\hat{a}(\mathrm{k}-1)}+\frac{\hat{b}}{\hat{a}}(\mathrm{k}=2,3, \ldots, \mathrm{n})
$$

The fifth step: In the initial conditions $\hat{\mathrm{x}}^{(1)}(1)=\mathrm{x}^{(1)}(1)=\mathrm{x}^{(0)}(1)$, Generate data series models are available:

$$
\hat{\mathrm{x}}^{(0)}(1)=\mathrm{x}^{(0)}(1), \hat{\mathrm{x}}^{(0)}(\mathrm{k})=\left(1-\mathrm{e}^{\hat{a}}\right)\left(\mathrm{x}^{(0)}(1)-\frac{\hat{b}}{\hat{a}}\right) \mathrm{e}^{-\hat{a}(\mathrm{k}-1)}, \mathrm{k}=2,3, \ldots, \mathrm{n}
$$

Namely, $\hat{\mathrm{x}}^{(0)}(1)=\mathrm{x}^{(0)}(1), \quad \hat{\mathrm{x}}^{(0)}(\mathrm{k})=\left(1-\mathrm{e}^{\hat{a}}\right)\left(\mathrm{x}^{(0)}(1)-\frac{\hat{b}}{\hat{a}}\right) \mathrm{e}^{-\hat{a}(\mathrm{k}-1)} \quad, k=2,3, \ldots, \mathrm{n}$

So, $k=2,3, \ldots, \mathrm{n}$ is substituted into the formula, getting the fitted values of the initial data; when $k>n$, to get the gray model predictive value for the future.

The sixth step: $Y^{(0)}=\left(\mathrm{y}^{(0)}(1), \mathrm{y}^{(0)}(2), \ldots, \mathrm{y}^{(0)}(\mathrm{n})\right), Z^{(0)}=\left(\mathrm{z}^{(0)}(1), \mathrm{z}^{(0)}(2), \ldots, \mathrm{z}^{(0)}(\mathrm{n})\right)$ repeat steps one to step five。

\subsection{Model accuracy test}

Gray model prediction test are generally residual test and posterior variance test.

First, the relative size of the error test method, it is a straightforward arithmetic test methods comparing point by point. In this method, prediction data is compared with the actual data, observing the relative error whether satisfies the practical requirements.

The actual data set is used for modeling:

$$
\begin{aligned}
& X^{(0)}=\left(\mathrm{x}^{(0)}(1), \mathrm{x}^{(0)}(2), \mathrm{x}^{(0)}(3), \ldots, \mathrm{x}^{(0)}(\mathrm{n})\right), \text { according to GM }(1,1) \text { modeling method to obtain } \\
& X^{(1)}=\left(\hat{\mathrm{x}}^{(1)}(1), \hat{\mathrm{x}}^{(1)}(2), \hat{\mathrm{x}}^{(1)}(3), \ldots, \hat{\mathrm{x}}^{(1)}(\mathrm{n})\right), \mathrm{X}^{(1)} \text { do a regressive generate transformed into } X^{(0)} \\
& , \text { Model value of actual data : } X^{(0)}=\left(\hat{\mathrm{x}}^{(0)}(1), \hat{\mathrm{x}}^{(0)}(2), \hat{\mathrm{x}}^{(0)}(3), \ldots, \hat{\mathrm{x}}^{(0)}(\mathrm{n})\right)
\end{aligned}
$$

Computing residuals, residual sequence is: 


$$
E=(e(1), \mathrm{e}(2), \ldots, \mathrm{e}(\mathrm{n}))=\mathrm{X}^{(0)}-\mathrm{X}^{(0)}
$$

where, $e(\mathrm{i})=\mathrm{x}^{(0)}(\mathrm{i})-\hat{\mathrm{x}}^{(0)}(\mathrm{i}), i=1,2, \ldots, \mathrm{n}$

Calculate the relative error, the relative error is :

$$
\varepsilon(\mathrm{i})=\frac{e(\mathrm{i})}{x^{(0)}(\mathrm{i})} \times 100 \%=\frac{x(\mathrm{i})-\hat{x}(\mathrm{i})}{x^{(0)}(\mathrm{i})} \times 100 \%
$$

$\varepsilon(\mathrm{i})=\frac{e(\mathrm{i})}{x^{(0)}(\mathrm{i})} \times 100 \%$ is the origin of the error , $\overline{\mathcal{E}}(\mathrm{i})=\frac{1}{m} \sum_{i=1}^{m}|\mathcal{E}(\mathrm{i})|$ is the average relative error of GM ( 1,1$)$ model; $p^{o}=(\varepsilon(\mathrm{k})-\bar{\varepsilon}) \times 100 \%$ is the model accuracy of $\operatorname{GM}(1,1)$ model , i.e., minimum error probability, and it generally requires $p^{o}>80 \%$, best $p^{o}>90 \%$.

Second, posterior deviation test belongs to the statistical concept, it is according to the probability distribution of residual test.

If the actual model data used was: $X^{(0)}=\left(\mathrm{x}^{(0)}(1), \mathrm{x}^{(0)}(2), \ldots, \mathrm{x}^{(0)}(\mathrm{n})\right)$, getting model value of actual data with the GM $(1,1)$ modeling method: $X^{(1)}=\left(\hat{\mathrm{x}}^{(1)}(1), \hat{\mathrm{x}}^{(1)}(2), \hat{\mathrm{x}}^{(1)}(3), \ldots, \hat{\mathrm{x}}^{(1)}(\mathrm{n})\right)$, Assuming variances of the actual data sequence $X^{(0)}$ and residuals sequence $\mathrm{E}$ separately are $^{S_{1}^{2} \text { and }} S_{2}^{2}$,

$S_{1}^{2}=\frac{1}{n} \sum_{i=1}^{n}\left(\mathrm{x}^{(0)}(\mathrm{i})-\overline{\mathrm{x}}^{(0)}\right), \bar{x}^{(0)}=\frac{1}{n} \sum_{i=1}^{n} x^{(0)}(\mathrm{i})$

$S_{2}^{2}=\frac{1}{n} \sum_{i=1}^{n}(\mathrm{e}(\mathrm{i})-\bar{e})^{2} \quad, \quad \bar{e}=\frac{1}{n} \sum_{i=1}^{n} e(\mathrm{i})$

calculating Posterior variance ratio :

$$
C=S_{2} / S_{1}
$$

Determine the model level, indicators such as Table 1:

Table 1. Model accuracy class

\begin{tabular}{|l|l|l|}
\hline Model accuracy class & Small Error probability P & Posterior inferior C \\
\hline I & $>0.95$ & $<0.35$ \\
\hline II & $>0.8$ & $<0.5$ \\
\hline III & $>0.7$ &, 0.65 \\
\hline IV & $\leq 0.7$ & $\geq_{0.65}$ \\
& & \\
\hline
\end{tabular}


Level Description: C values as small as possible, i.e., $S_{1}$ is much smaller than the ${ }^{S_{0}}$.raw date discrete is large, prediction error discrete is small, and prediction accuracy is high; $\mathrm{P}$ bigger the better, equally error probability is small, fitting accuracy high. If the residual test, posterior variance test can be passed, it can be predicted by their model, otherwise corrected residuals.

Table 2. Target prediction location

\begin{tabular}{|l|l|l|l|}
\hline Target prediction & Small error probability & Posterior variance ratio & Model accuracy \\
\hline$X$ & 1 & 0.188 & I \\
\hline$Y$ & 1 & 0.107 & I \\
\hline$Z$ & 1 & 0.115 & I \\
\hline
\end{tabular}

\subsection{Track forecast results and analysis}

The radius, velocity and range angle $\beta$ of observation point $\mathrm{D}$ are known, the plane parallel to the direction of velocity and passed point $\mathrm{D}$ can be approximated as missile flight plane, plane $Q_{2}$ that through geocentric $O$, point D and perpendicular to the direction of the bullet speed, plane $Q_{3}$ that through geocentric $O$, placement $\mathrm{B}$ and perpendicular to the direction of the bullet speed. Angle between plane $Q_{2}$ and plane $Q_{3}$ is the range angle $\beta$. Release impact coordinate, shown in FIG 1.

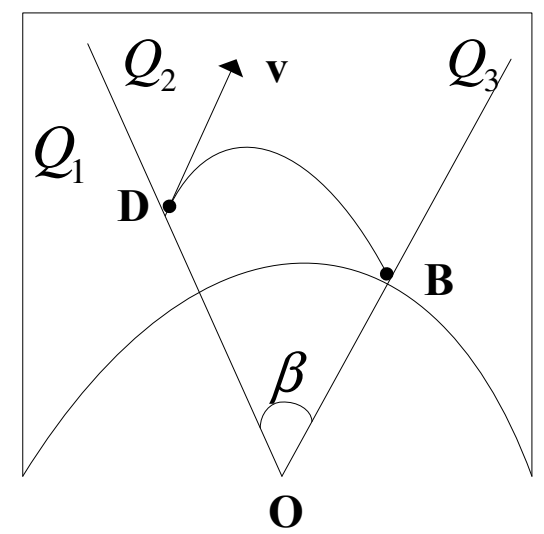

Figure 2. Solving placement schematic

If plane $Q_{1}$ is $A_{1} x+B_{1} y+C_{1} z=0$, normal vector is $n_{1}=A_{1} i+B_{1} j+C_{1} k \quad$, and $n_{1}=r_{O D} \times v=\left[\begin{array}{ccc}i & j & k \\ x_{0} & y_{0} & z_{0} \\ v_{x} & v_{y} & v_{z}\end{array}\right], i, j, k$ are unit vector for each axis, the flat $Q_{1}$ can be solved by the equation. 
If plane $Q_{2}$ is $A_{2} x+B_{2} y+C_{2} z=0$, normal vector is $n_{2}=A_{2} i+B_{2} j+C_{2} k$, and $n_{2}=r_{O D} \times n_{1}=\left[\begin{array}{ccc}i & j & k \\ x_{0} & y_{0} & z_{0} \\ A_{1} & B_{1} & C_{1}\end{array}\right]_{\text {, the flat }} Q_{2}$ can be solved by the equation.

If plane $Q_{3}$ is $A_{3} x+B_{3} y+C_{3} z=0$,normal vector is $n_{3}=A_{3} i+B_{3} j+C_{3} k \quad$, and $\left\{\begin{array}{c}n_{2} \square n_{3}=\left|n_{2}\right|\left|n_{3}\right| \cos \beta \\ n_{3} \square n_{1}=0 \\ \left|n_{3}\right|=1\end{array}\right.$

, the flat $Q_{3}$ can be solved by the equation, intersection of the plane $Q_{1}$, the plane $Q_{3}$ and the earth's surface is the placement of $\mathrm{B}, B=(x, y, z)$, and $\left\{\begin{array}{c}A_{1} x+B_{1} y+C_{1} z=0 \\ A_{3} x+B_{3} y+C_{3} z=0 \\ x^{2}+y^{2}+z^{2}=R^{2}\end{array}\right.$

Placement coordinates can be obtained by solve formulas, after forecast, Placement coordinates is $(-2423400,-2103400,5488000)$, error radius $1000 \mathrm{~m}$, algorithm complexity $0(n)$.

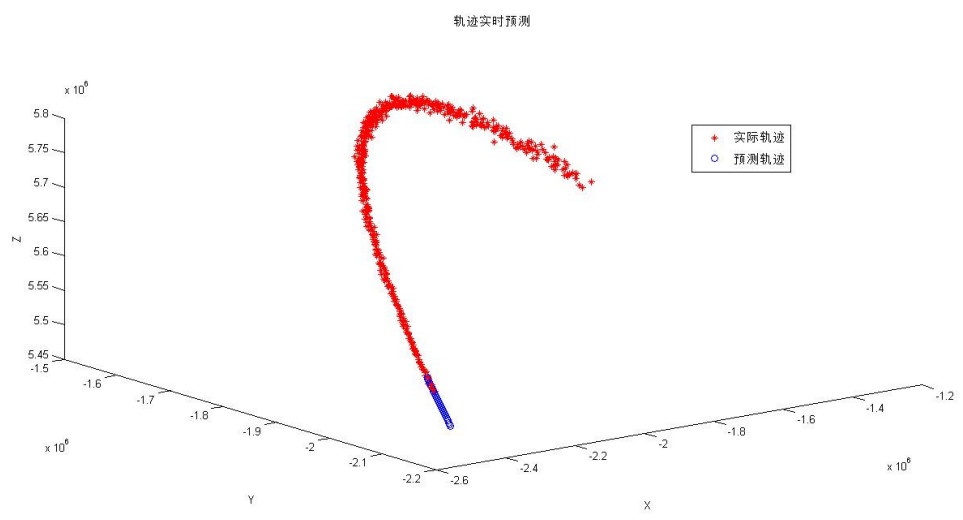

Figure 3. Forecasting model predicted target track

\section{REFERENCES}

[1] Li Feng, Jin Hongbin, Ma Jianchao. A New Method of Coordinate Transformation Under MultiRadar Data Processing System [J].Control \& Measurement, 2007,23(4):303-305.

[2] Sun Fuming. Research on State Estimation and Data Association of Motion Targets [D]. University of Science and Technology of China,2007. 
International Journal on Cybernetics \& Informatics (IJCI) Vol. 4, No. 1, February 2015

[3] Song Yingchun. Research on Kalman Filter in Kinematic Positioning [D].Central South University, 2006.

[4] Liu Na. Study on Data Association Method in Multitarget Tracking of Ballistic Missile Denfense Radar [D]. National University of Defense Technology, 2007.

[5] Feng Yang .The Research on Data Association in Multi-target Tracking [D].XiDian University,2008.

[6] Zhou Hongren. Maneuvering target "current" statistical model and adaptive tracking algorithm [J]. Journal of Aeronautics, 1983,01:73-86

[7] Yao Jinjie. Research on Techniques of Target Localization based Stations[D].North University of China,2011.

[8] Liu Gang. Multi target tracking algorithm research and Realization [D]. Northwestern Polytechnical University,2003.

\section{Authors}

Qing-ping $\mathbf{Y u}$ is currently studying in Mechanical and Electronic Engineering from Shanghai University of Engineering Science, China, where she is working toward the Master degree. Her current research interests include ant colony algorithm, their design and develop in Embedded system.

Xiao-Ming You received her M.S. degree in computer science from Wuhan University in 1993, and her Ph.D. degree in computer science from East China University of Science and Technology in 2007. Her research interests include swarm intelligent systems, distributed parallel processing and evolutionary computing. She now works in Shangha i University of Engineering Science as a professor.
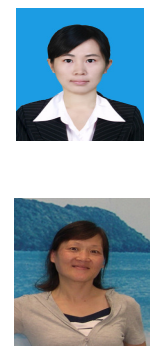